\title{
TOLERANCIA TERMICA DE ALGUNOS PECES MARINOS TROPICALES: ESTUDIO PRELIMINAR
}

\author{
K. S. CHUNG \\ Instituto Oceanográfico \\ Universidad de Oriente, Cumaná, Venezuela
}

\section{SYNOPSIS}

The critical thermal maximum (CTM) and the first equilibrium loss (FEL) of six tropical marine fishes were determined. CTM' were Mugil curema $\left(41.4^{\circ} \mathrm{C}\right.$ ), Gerres $s p$. $\left(39.2^{\circ} \mathrm{C}\right)$, Orthopristis ruber $\left(38.7^{\circ} \mathrm{C}\right)$, Archosargus rhomboidalis $\left(39.39^{\circ} \mathrm{C}\right)$, Gobioides sp. $\left(38.6^{\circ} \mathrm{C}\right)$, and \begin{tabular}{l} 
and Chilomycterus sp. $\left(38.3^{\circ} \mathrm{C}\right)$. The mean difference between $\mathrm{CTM}$ and $\mathrm{FEL}$ ranged from 0.93 to $2.66^{\circ} \mathrm{C}$ and was significantly different. \\
\hline
\end{tabular}

\section{Introduccion}

La temperatura actúa de varias formas sobre los diferentes organismos; puede matar, reducir la actividad, interactuar con otros factores ambientales tales como salinidad, $\mathrm{pH}$, oxigeno metales pesados etc, limitar la reproducción y ser un agente canalizador cuando existe un gradiente (Brett, 1960).

El máximo o mínimo termico critico (MTC) es la media aritmetica del punto térmico al cual la actividad locomotora comienza a desorganizarse y el animal pierde su habilidad para escapar de condiciones que prontamente lo conducirian a su muerte, cuando son calentados o enfriados a partir de una temperatura de aclimatación previa, a una tasa constante de variación, que permita a la temperatura corporal seguir la temperatura ambiental del experimento sin que ocurra un retardo significativo en el tiempo (Cowles \& Bogert, 1944: Lowe \& Vance, 1955; Hutchinson, 1961), Lowe \& Heath (1969) han aplicado este concepto (MTC) a peces como un criterio de tolerancia térmica. Recientemente la prímera pérdida de equilibrio (PPE) tambien ha sido usado como un criterio de tolerancia térmica (Cox, 1974; Copleland et al., 1974).

Hay poca información disponible sobre el MTC y la PPE de peces marinos tropicales. El objetivo de este estudio fue deter. minar el MTC y la PPE de seis especies de peces marinos tropicales como un criterio de tolerancia de temperatura.

\section{Materiales y Metodos}

Los seis peces marinos tropicales identificados la lisa (Mugi) curema), la mojarra (Gerres sp)*, el corocoro (Orthopristis ruber), la cagalona (Archogargus rhomboidalis), los gobidos (Gobioides $\mathrm{sp})^{*}$ y el tamborin (Chilomycterus sp)* fueron colectados de playas vecinas a Turpialito (Golfo de Cariaco). La temperatura del agua donde se capturaron los ejemplares fue un $26^{\circ} \mathrm{C}$. Los peces fueron mantenidos en acuarios de $(30 \times 40 \times 80 \mathrm{~cm})$ por un dia bajo condiciones de laboratono $\left(26 \pm 1{ }^{\circ} \mathrm{C}\right)$. El promedio de la longitad standard y el numero de peces estudiados en cada especie fueron: la lisa $(42 \mathrm{~mm}, 10)$; la mojarra $(52 \mathrm{~mm}, 10)$; el corocoro $(5 \mathrm{~mm}, 10)$; los gobidos (34 mm, 10); el tamborin $(138 \mathrm{~mm}, 3)$; y la cagalona (129 mm, 3).

El máximo térmico crítico (MTC) y la primera pérdida de equilibrio (PPE) fueron determinados, colocando de 1 a 3 peces en un frasco con dos litros de agua de mar natural y una piedra de aireación. El frasco fue calentado por tres calentadores de acuario con un transformador de voltaje. Las tasas de elevación de temperatura a las cuales los peces marinos fueron sometidos fue de $1^{\circ} \mathrm{C} / \mathrm{min}$. Las temperaturas a las cuales se manifestaron la primera pérdida de equilibrio (PPE) y el cese de pulsaciones del operculo (MTC) fueron selecionados como punto final.

\section{Resultados y Discusion}

La primera pérdida de equilibrio (PPE) y el máximo térmico crítico (MTC) como criterio de tolerancia térmica y la diferencia entre la temperatura de aclimatación y el MTC estan representados en la Tabla I. Los intervalos de valores de PPE y de MTC son 36.0 a $40.7^{\circ} \mathrm{C}$ y 38.6 a $41.5^{\circ} \mathrm{C}$, respectivamente. La diferencia entre la temperatura de aclimatación (ambiente) y el MTC osciló entre 11.6 y $13.5^{\circ} \mathrm{C}$. Por lo tanto los organismos estudiados pueden

\footnotetext{
* En estudio para su identificación.
}

tolerar $11^{\circ} \mathrm{C}$ por encima de la temperatura ambiente. Los valores obtenidos para MTC indican que las especies mas resistentes estan en el seguiente orden: la lisa, la mojarra, el corocoro, la cagalona, los gobidos y el tamborin.

Tabla I - La primera pérdida de equilibrio (PPE) y el máximo térmico crítico (MTC) expresados en ${ }^{\circ} \mathrm{C}$, como un criterio de tolerancia térmica y la diferencia entre la temperatura de aclimatación y la de MTC (D), de seis peces marinos tropicales colectados a $26^{\circ} \mathrm{C}$.

\begin{tabular}{lccc}
\hline Especie & PPE $\left({ }^{\circ} \mathrm{C}\right)$ & MTC $\left({ }^{\circ} \mathrm{C}\right)$ & D $\left({ }^{\circ} \mathrm{C}\right)$ \\
\hline \hline Mugil curema & 40.7 & 41.5 & 13.5 \\
Gerres sp. & 38.5 & 39.2 & 12.2 \\
Orthopristis ruber & 37.2 & 38.7 & 11.7 \\
Archosargus rhomboidalis & 38.7 & 39.9 & 12.2 \\
Gobioides sp. & 36.0 & 36.6 & 11.6 \\
Chilomycterus sp. & 38.3 & 39.3 & 12.3 \\
\hline
\end{tabular}

El análisis de varianza indica que tos vajores del MTC de seis peces estudiados son significativamente diferentes a un nivel de 1\% (Tab. II). Para determinar que grupo o grupos de MTC fueron significativamente diferentes, se aplicó una prueba de Duncan (Tab. II). La comparación multiple de medias indicó que la toleran-

Tabla II - Análisis de varianza del máximo térmico critico (MTC) de seis peces marinos tropicales capturados a $26^{\circ} \mathrm{C}$ y prueba de Duncan los MTC no conectados son significativamente diferente a un nivel de $1 \%$.

\begin{tabular}{lcccc}
\hline & \multicolumn{4}{c}{ ANOVA } \\
\cline { 2 - 5 } Fuente & G1 & SC & MG & FS \\
Especies & 5 & 59.52 & 11.90 & $51.73^{* *}$ \\
Error & 40 & 9.41 & 0,23 & \\
Total & 45 & 68.93 & &
\end{tabular}

\section{PRUEBA DE DUNCAN}

\begin{tabular}{|c|c|c|c|c|c|c|}
\hline \multirow[t]{2}{*}{$\begin{array}{l}\text { Especies* } \\
\text { MTC }\end{array}$} & $\begin{array}{r}\mathrm{Gb} \\
38.6\end{array}$ & $\begin{array}{l}\text { Or } \\
38.7\end{array}$ & $\begin{array}{c}\mathrm{Ge} \\
39.2\end{array}$ & $\begin{array}{c}\text { Ar } \\
39.3\end{array}$ & $\begin{array}{c}\text { Ch } \\
39.9\end{array}$ & $\begin{array}{r}\text { Mc } \\
41.5\end{array}$ \\
\hline & & & & & & \\
\hline $\begin{array}{l}\mathrm{Gb}, \text { Go } \\
\mathrm{Or}, \text { Ort } \\
\mathrm{Ge}, \text { Ger } \\
\mathrm{Ar}, \text { Arc } \\
\mathrm{Ch}, \mathrm{Ch} \\
\mathrm{Mc}, M u\end{array}$ & $\begin{array}{l}\text { sp. } \\
\text { is ruber } \\
\text { us rhom } \\
\text { erus sp. } \\
\text { ma }\end{array}$ & oidalis & & & & \\
\hline
\end{tabular}


cia a la temperatura en la lisa fue significativamente diferente a la de otras especies estudiados. El MTC del tamborin fue también significativamente diferente del MTC de los gobidos estudiados. La comparación multiple de medias representada en la Tabla II, confirma que la lisa es la especie que tolera una mayor temperatura entre las seis especies estudiadas y puede tolerar $13.5^{\circ} \mathrm{C}$ por encima de la temperatura ambiente.

El MTC $\left(39.1^{\circ} \mathrm{C}\right)$ de Micropogon undulatus aclimatado a $28^{\circ} \mathrm{C}$ (Copleland et al., 1974) y el MTC $\left(39.24^{\circ} \mathrm{C}\right)$ de Lepomis racrochirus aclimatado a $26^{\circ} \mathrm{C}(\mathrm{Cox}, 1974)$ fueron similares los MTC (38.6-39.9० C) de los seis peces estudiados, excepto para la lisa en la cual fue de $41.5^{\circ} \mathrm{C}$. Hassan \& Spotila (1976) encontraron que el MTC de larvas de Esox masquinogy aclimatados a $25^{\circ} \mathrm{C}$ fuctua entre 33.2 y $36.1^{\circ} \mathrm{C}$. Esto indica que estas larvas son mucho mes sensibles al calor que los peces juveniles y adultos. El MTC $\left(41.5^{\circ} \mathrm{C}\right.$ ) de la lisa estudiada es también mas alto que la $\mathrm{TL}_{50}$ (temperatura letal) a 3 horas y a una temperatura de $39^{\circ} \mathrm{C}$ viviendo ésta en el canal de salida de una planta electrica en el Estado Texas (USA) durante Junio y Septiembre (1974-1975) cuando la temperatura del agua del canal de entrada era $26-28^{\circ} \mathrm{C}$ (Chung, 1977).

Las diferencias de promedio entre el MTC y la PPE oscilaron entre 0.93 a $2.66^{\circ} \mathrm{C}$. Para determinar si las diferencias entre MTf y PPE eran significativos, se aplicó una prueba del t - dependiente (Tab. III). Esta prueba indica que en todos los peces estudiados hay una diferencia significativa entre MTC y PPE. La infor-

Tabla III - Resultados de la prueba del t-dependiente entre la primera pérdida de equilibrio (PPE) y el máximo térmico critico (MTC) de seis peces marinos tropicales colectados a $26^{\circ} \mathrm{C}, \overline{\mathrm{d}}$ : diferencia de las medias entre MTC y PPE; Sd: desviación standard de la diferencia de las medias).

\begin{tabular}{lllll}
\hline Especie & GI & $\bar{d}$ & S $\bar{d}$ & Valor de t \\
\hline \hline Mugil curema & 9 & 1.30 & 0.0076 & $171.05^{* *}$ \\
Gerres sp. & 9 & 0.93 & 0.00725 & $12.75^{* *}$ \\
Orthopristis ruber & 9 & 1.48 & 0.0458 & $32.20^{* *}$ \\
Archosargus rhomboidalis & 2 & 1.03 & 0.0216 & $47.68^{* *}$ \\
Gobioides sp. & 9 & 2.66 & 0.0400 & $66.50^{* *}$ \\
Chilomycterus sp. & 2 & 1.00 & 0.0633 & $15.79^{* *}$ \\
\hline
\end{tabular}

** : Significativo a un nivel de $1 \%$. mación antes señalada confirma que se requiere la mayor temperatura para alcanzar el punto final (MTC) del movimiento opercular despues que el pez ha alcanzado la primera pérdida de equilibrio (PPE).

\section{Agradecimiento}

Deseo expresar mi agradecimiento a los Professores Taizo Okuda y Andrés Lemus por la ayuda prestada en la realización de este trabalho. Tambien, se expresa agradecimiento a la Organización de los Estados Americanos por ayudar en parte este estudio. Al Profesores Aida Martínez y Gregorio Reyes por su colaboración en la revisión del manuscrito. Al sr. Jorge Hernández por su ayuda en el trabajo de campo y de laboratorio.

\section{Referencias Bibliográficas}

BRETT, J.R. 1960. Thermal requirements of fish. Three decades of study, 1940-1970. In: Tarzwell, C.M., ed. - Biological problems in water pollution. Ohio, Public Health Service, p. $110-117$.

CHUNG, K.S. 1977. Heat resistance of crustaceans and fishes taken from the intake canal of an estuarine power plant and their predicted survival in the discharge canal. Ph. D. Dissertation. Texas A \& M University, 443 p.

COPLELAND, B.J.; LANEY, R.W. \& PENDLETON, E.C. 1974. Heat influences in estuarine ecosystems. In: Gibbons, J. W. \& Sahritz, R. R., ed. - Thermal ecology. U. S. Atomic Energy Commission, Tech. Inform. Center, p. 423437.

COWLES, R.B. \& BOGERT, C.M. 1944. A preliminary study of the thermal requirements of desert reptiles. Bull. Am. Mus. nat. Hist., 83: 265-296.

COX, D.K. 1974. Effects of three heating rates on the critical thermal maximum of bluegill. In: Gibbons, J.W. \& Sharitz, R.R., ed. - Thermal ecology. U.S. Atomic Energy Commission, Tech. Inform. Center, p. 158-163.

HASSAN, K.C. \& SPOTILA, J.R. 1976. The effect of acclimation on the temperature of young muskellunge fry. In: Esch, G.W. \& McFarlane, R.W., ed. - Thermal ecology II. Energy Res. Development Admin., Tech. Inform. Center, p. 136-140.

HUTCHINSON, V.H. 1961. Critical thermal maxima in salamanders. Physiol. Zool., 34: 92-125.

LOWE, C.H. \& HEATH, W.G. 1969. Behavioral and physiological responses to temperature in the desert pupfish (Cyprinodon macularius). Physiol. Zool., 43: 35-59.

- _ - \& VANCE, V,J. 1955. Acclimation of the critical thermal maximum of the reptile, Urosaurus ornatus. Science, 122: 73-74. 